Andreas Münzmay*

\title{
Lesen und Schreiben im digitalen Dickicht
}

\author{
Musikwissenschaft, Digital Humanities und die hybride Musikbibliothek
}

https://doi.org/10.1515/bfp-2018-0031

Zusammenfassung: Digitalisierung generiert ,hybride“ Objekte, die zwar als digitale Datei lesbar und manipulierbar sind, das zugrundeliegende kulturhistorische Objekt jedoch weiterhin ,enthalten'. Eine Bibliothek, die Objekte aus ihren Beständen digitalisiert, begründet also eigentlich keine sog. digitale Bibliothek, sondern eine hybride Bibliothek. Im Falle musikalischer Überlieferung ist die Sachlage aufgrund der medienhistorischen Komplexität musikalischer Artefakte besonders unübersichtlich. Verschärft wird die heutige Situation noch durch die Erzeugnisse der Digital Humanities, die keineswegs bloß ,Informationen' sind, sondern ihrerseits komplexe und in der Regel hybride Textgebilde, die häufig komplette digitalisierte Re-Publikationen von Kulturobjekten einbinden, die digital ,beschriftet' und vernetzt werden. Das digitale ,Dickicht' erfordert einen gleichsam genetischen und relationalen Katalog, in dem die konkreten kulturhistorischen Objekte in ihren konkreten Sammlungskontexten - also als Unikate-Ankerfunktion haben. ${ }^{1}$

Schlüsselwörter: Hybride Objekte; Digitale Bibliothek; Digital Humanities; Metadaten; Katalog

\section{Reading and Writing in the Digital Thicket: Musicology, Digital Humanities, and the Hybrid Music Library}

Abstract: Digitization produces ,hybrid‘ objects that are readable as digital data files but at the same time still ,contain' the original cultural-historical object. In this perspective, ,digital collections', ,digital libraries', etc. are in fact hybrid collections, or libraries. For musical artefacts, the range of historical media is especially complex, which again concerns the digital world. Digital Humanities publications also contribute to the complex and confusing situation as they themselves are not only ,information', but as a rule, complex and hybrid textual systems that often contain

1 Der Essay geht zurück auf einen Vortrag bei der AIBM-Jahrestagung, Symposium der AG Musikabteilungen an wissenschaftlichen Bibliotheken, Detmold, 8. September 2016.

*Kontaktperson: Prof. Dr. Andreas Münzmay, andreas.muenzmay@uni-paderborn.de complete digitized re-publications of cultural objects, providing them with digital ,inscriptions', and interlinking them. So the hybridity of ,digital libraries' increases as soon as Digital Humanities publications are integrated. Therefore, metadata should mirror precisely the hybrid nature of the digitized/digital objects catalogued.

Keywords: ,Hybrid‘ objects; digital library; digital humanities; metadata, catalogue

Inhalt

1 Vom Labyrinth des Wissens ins Dickicht der Hybride ................... 236

2 Hybride lesen - eine Hauptbeschäftigung (digitaler) historischer Kulturwissenschaften . . . . . 238

3 Noch hybridere Hybride schreiben - eine Hauptbeschäftigung von Digital Humanities . . . . . . 243

4 Potenziert hybride Bestände . . . . . . . . . . . . 245

\section{Vom Labyrinth des Wissens ins Dickicht der Hybride}

Umberto Eco erinnerte 1984 in seiner Semiotica e filosofia del linguaggio ${ }^{2}$ daran, dass seit Diderot und d'Alembert, den Enzyklopädisten der Aufklärung, der Diskurs über Wissensorganisation, wie er für die Konzeptionen von Enzyklopädien, aber auch von Bibliotheken grundlegend ist, „von einer zugrundeliegenden Metaphysik oder einer Metapher (oder einer Allegorie) regiert wird: der Idee des Labyrinths. “"

Schon 1980 hatte Eco die Labyrinth-Metapher wirkungsvoll popularisiert: Die Hauptrolle im Roman Der Name der Rose spielt eine mittelalterliche Klosterbibliothek, in der es das Manuskript des verschollenen zweiten Buchs von

2 Eco, Umberto: Semiotica e filosofia del linguaggio. Turin 1984. Der betreffende Abschnitt ist unter dem Titel „Die Enzyklopädie als Labyrinth“ in dt. Übers. abgedruckt in Uwe Wirth (Hg.): Kulturwissenschaft - Eine Auswahl grundlegender Texte. Frankfurt/M. 2008, 262-67.

3 Eco (Anm. 2), dt. zit. nach Wirth (Anm. 2) 262. 
Aristoteles' Poetik (das Buch über die Komödie) zu finden gilt. Die Bibliothek gestaltet Eco als labyrinthische, komplizierte Verzweigungen aufweisende, insgesamt aber systematisch geschlossene Universalbibliothek. Das verbotene, giftige Manuskript wird von einem blinden Greis namens Jorge $^{4} \mathrm{im}$ „finis Africae“ (einem geheimen, nur auf genau einem Weg erreichbaren Raum) gleichsam als der Minotaurus dieses Irrgartens unter Verschluss gehalten. Allerdings lässt Eco diese Bibliothek, nachdem sie samt ihrer Sollbruchstelle (das nicht einzuordnende, nicht systemkonforme Manuskript) durch die Detektive (Spurensucher, Leser) Guglielmo und Adson entschlüsselt ist, unwiederbringlich in einer Feuersbrunst untergehen: „Dann ertönte ein dumpfes Krachen: Der Boden des Labyrinths hatte nachgegeben, brennende Balken stürzten in das darunterliegende Stockwerk". ${ }^{5}$ Der Zusammenbruch der Tragkonstruktion der überkommenen Wissensordnung reißt unerbittlich alle Lebensbereiche mit sich: Studierzimmer, Küche, Dormitorum und schließlich die Kirche selbst. Die Neuzeit dämmert.

Wissen sei, so präzisiert Eco dann in der Semiotik, tatsächlich gerade nicht als geschlossenes, kartografierbares Gebilde konzeptualisierbar, sondern allenfalls als ein „Labyrinth der dritten Art“, bei dem „wir es mit einem Netz zu tun“ haben. Darin könne ,jeder Punkt mit jedem anderen Punkt verbunden werden [...], und wo die Verbindungen noch nicht entworfen sind, können sie trotzdem vorgestellt" werden." (Ein solches Netz ist also ein vierdimensionales, auch die zeitliche Komponente einschließendes Gebilde mit wenig Gewissheiten und vielen Möglichkeiten.) Schon Diderot und d'Alembert hätten dies im Prinzip erkannt und daher die hierarchische Systematik der Encyclopédie durch ein neuartiges Querverweissystem ergänzt. „Das beste Bild eines Netzes“ aber biete, so Eco weiter, „die pflanzliche Metapher des Rhizoms, die Deleuze und Guattari (1976) vorschlagen, "6 also ein anti-genealogisches, unendlich in sich verknotetes, beständig veränderbares Gebilde ohne definierte Außengrenzen, so dass niemand ,eine globale Beschreibung des ganzen Rhizoms liefern" kann. ${ }^{7}$ Eine solche Struktur sei, wie Pierre

4 Ein Hinweis auf Jorge Luis Borges, der in seiner 1941 erstveröffentlichten Erzählung La biblioteca de Babel eine auf den unendlichen Kombinationsmöglichkeiten des Alphabets beruhende fantastische Universalbibliothek entwarf, die dadurch allumfassend und unlesbar zugleich ist.

5 „A quel punto si udì uno schianto: il pavimento del labirinto aveva ceduto in qualche punto precipitando le sue travi infuocate al piano inferiore“. Eco, Umberto: Il nome della rosa. 47. Aufl. Mailand 2001, 491; dt. Übers. von Burkhard Kroeber zit. nach Umberto Eco: Der Name der Rose, 7. Aufl. München 1986, $620 \mathrm{f}$.

6 Eco (1984, Anm. 2), dt. zit. nach Wirth (Anm. 2) 263.

7 Ebd. 264.
Rosenstiehl es im Zuge mathematischer Nachweise treffend genannt habe, zudem „kurzsichtig“: 8

„An keinem seiner Knoten kann man die globale Ansicht aller Möglichkeiten haben [...], sondern nur die lokale Ansicht der am nächsten gelegenen: jede lokale Beschreibung des Netzes ist eine Hypothese des weiteren Verlaufs und kann falsch sein; in einem Rhizom ist Blindheit die einzige Art des Sehens (lokal), und Denken heißt, nach dem Weg zu tasten. “9

Das heutige World Wide Web konnten Rosenstiel, Deleuze, Guattari und Eco schwerlich vorhersehen. Aber doch schwingt die für die 1970er-Jahre prägende technische und theoretische Informatisierung (symptomatisch z.B. die Entstehung der akademischen Fächer Informatik und Computerlinguistik) im eben angerissenen Diskurs mit, was vielleicht zumindest ein Stück weit erklärt, dass die seinerzeit mit poststrukturalistisch-systemkritisch-postmoderner Verve als zentral erkannten Faktoren ,kein Außen', Unvorhersagbarkeit, Veränderlichkeit, Verbindungsreichtum, NichtHierarchizität auch für das enzyklopädisch-universale digitale Wissens- und Informationsnetz WWW so präzise zu passen scheinen..$^{10}$ Es könnte demnach ein „Rhizom“sein.

Gleichwohl spreche ich versuchsweise lieber vom digitalen „Dickicht“. Warum denn noch eine Metapher? Das Rhizom scheint mir trotz allem noch zu uniform, wird es doch von einer Pflanze oder einem Pilz einer Art gebildet. Anders das artenreiche Dickicht: Im Dickicht kann alles vorkommen (auch Rhizome, auch Bäume); in ihm kann alles ineinander verwachsen, in Konkurrenz treten, sich verschlingen, verdrängen, bündeln und verstärken. Je nach Standort werden Substrukturen, Lichtungen, Inseln, Stockwerke (oder mit Deleuze und Guattari gesprochen: „Plateaus “"11) erkennbar.

Das Metaphern-Experiment ist hauptsächlich durch drei Eindrücke provoziert. Zum einen scheinen im Zuge der Digitalisierung, gerade auch unter der Flagge von „Digital Humanities“, Bäume (eindeutige, streng hierarchische Strukturen) eine neue Konjunktur zu erleben - man denke etwa an Normdaten, Ontologien, Authority Control und Ähnliches. Zum Zweiten modert und verrottet im Dickicht stets irgendwo irgendetwas vor sich hin, oft unbemerkt zurecht wird das Nachhaltigkeitsproblem gerade auch im Kontext digitaler Informationskulturen thematisiert. Im

8 Eco bezieht sich auf Rosenstiehl, Pierre: Labyrinthologie mathématique. In: Mathématiques \& sciences humaines, Bd. 33 (1971) 5-32. 9 Eco (1984, Anm. 2), dt. zit. nach Wirth (Anm. 2) 264.

10 Wobei es selbstredend noch andere entscheidende Charakteristika aufweist, wie etwa die prinzipielle Adressierbarkeit und damit blitzschnell-sprungartige Aufsuchbarkeit jedes beliebigen Knotens. 11 Deleuze, Gilles; Guattari, Felix: Mille plateaux. Paris 1980. 
Zentrum soll im Folgenden aber ein dritter Aspekt stehen: Das digitale Dickicht mit einer Reinkultur zu verwechseln, nur weil alles darin Datei, Bits und Bytes, Null und Eins ist, wäre äußerst kurzsichtig. Vielmehr sind als eine gleichsam natürliche Folge der Digitalisierung die Materiallandschaften, in welchen man sich auf irgendeiner Spur irgendeiner (Musik-)Kultur bewegt, um etwas herauszufinden und zu verstehen, ganz maßgeblich von Hybriden bewachsen. Auch die Hybride aber sind im Dickicht metaphorisch viel besser unterzubringen als etwa im Rhizom.

Digitalisierung bringt grundsätzlich Hybride hervor. Denn das originale, dem informationstechnischen Vorgang Digitalisierung unterzogene musikkulturelle Objekt - eine Partitur, ein Brief, eine Schallplatte, ein Libretto, ein Bild, ein Druck, eine Handschrift, ein Buch, ein Aufsatz, eine Zeitung, was auch immer - verschwindet ja durch Digitalisierung nicht einfach oder mutiert einfach nur zu Kode, zur Datei. Es bleibt gedanklich vielmehr im Digitalisat gleichsam enthalten, mit allen seinen potenziellen Gebrauchsmustern, Regeln, Geschichten, mit seiner Abundanz oder Seltenheit, seiner Bekanntheit oder Obskurität, seiner Neuheit oder Abgenutzheit, seinem Bestandskontext und seinen Gebrauchsspuren, und nicht zuletzt auch mit seinen materialen Eigenschaften (die Maße, der Einband, die Ausrisse und Einklebungen, die Schreibschichten und -materialien etc.). ${ }^{12}$ Die Datei ist aber auch nicht einfach nur ein Abbild oder ,Stellvertreter' des Objekts, sondern zugleich selbst ein neues kulturhistorisches Objekt eigenen Rechts. Dies sieht man zum Beispiel daran, dass es ganz anders handzuhaben ist, oder daran, dass an ihm infolge digitaler Auswertungsverfahren spezifische Diskurse ansetzen können, die am Original so gar nicht ansetzen könnten, oder auch daran, dass ein kulturelles Artefakt infolge seiner Digitalisierung in einen, oft mehrere zusätzliche Sammlungskontexte eingeführt wird. ${ }^{13}$ In Anbetracht von René Magrittes berühmter in Öl gemalter und mit dem Satz „Ceci n'est pas une pipe“ konfrontierter Pfeife ${ }^{14}$ müsste man somit für

12 Siegert, Christine: Wissenschaftliches Arbeiten im Zeitalter digitaler Quellen. In: Zeitschrift für Bibliothekswesen und Bibliographie 59 (2012), 158-65. DOI: 10.3196/1864295012593467.

13 Etwa ein Libretto der in der Bayerischen Staatsbibliothek München aufbewahrten „Sammlung Her“, das als Digitalisat auch Teil einer „Libretto-Portal“ genannten Meta-Sammlung ist, in die Europeana Collections Eingang fand und zum Googlebook wurde. Dass aber das Librettoexemplar auch in digitalisierter Form nur dann vollständig gelesen/verstanden werden kann, wenn man es als ein in der Sammlung Her kontextualisiertes Objekt begreift und ernst nimmt, begründet seine Hybridität.

14 Magritte, René: La trahison des images (Ceci n'est pas une pipe), Öl auf Leinwand, 1929. Los Angeles County Museum of Art, Digitalisat unter http://collections.lacma.org/node/239578.
Digitalisate betonen: Die digitalisierte Pfeife ist nicht nur ,keine Pfeife', sondern zugleich auch ,mehr als die Pfeife‘

These 1: Da Digitalisierung keine ,rein digitalen', sondern prinzipiell hybride Objekte generiert, begründet eine (Musik-)Bibliothek, die Objekte aus ihren Beständen digitalisiert und den Lesern zur Verfügung stellt, niemals einfach eine „digitale Bibliothek“ oder „digitale Sammlung“ (so eingängig solche Formeln auch sein mögen), sondern stets eine hybride (Musik-)Bibliothek.

\section{Hybride lesen - eine Hauptbeschäftigung (digitaler) historischer Kulturwissenschaften ${ }^{15}$}

Normal sind im Dickicht der Hybride solche Erscheinungen:

Blaise, Adolphe Benoît

Annette et Lubin - Mus.3143-F-3 - [ Partitur ]

\section{Medientyp: E-Book}

Titel: Annette et Lubin - Mus.3143-F-3

Beteiligte: Blaise, Adolphe Benoît

Erschienen: [S.I.], [1765]

Ausgabe: [Partitur]

Enthält: Annette et Lubin / Blaise, A.B

Ballets / Anonymus

Umfang: Abschrift

Reproduktionsreihe: Königliche Privat-Musikaliensammlung digital

Digitale Sammlungen / Sächsische Landesbibliothek - Staats- und Universitätsbibliothek Dresden : Musik

Art der Reproduktion: Online-Ausg.

Ort der Reproduktion: Dresden

Reproduktion durch: SLUB, 2011

Standort des Originals: SLUB Dresden

Schlagwörter: Quelle

Beschreibung: Kurzaufnahme einer Musikhandschrift.

Abb. 1: http://katalogbeta.slub-dresden.de/id/0012704209/\#detail (Permalink), Screenshot (Ausschnitt, 25.8.2017)

15 „Digitale historische Kulturwissenschaften“ schlägt Schmale als präziseres Komplement zu „Digital Humanities“ vor: Einleitung. Digital Humanities - Historische Kulturwissenschaften, s. Schmale, Wolfgang (Hg.): Digital Humanities. Praktiken der Digitalisierung, der Dissemination und der Selbstreflexivität. Stuttgart 2015, 9. 


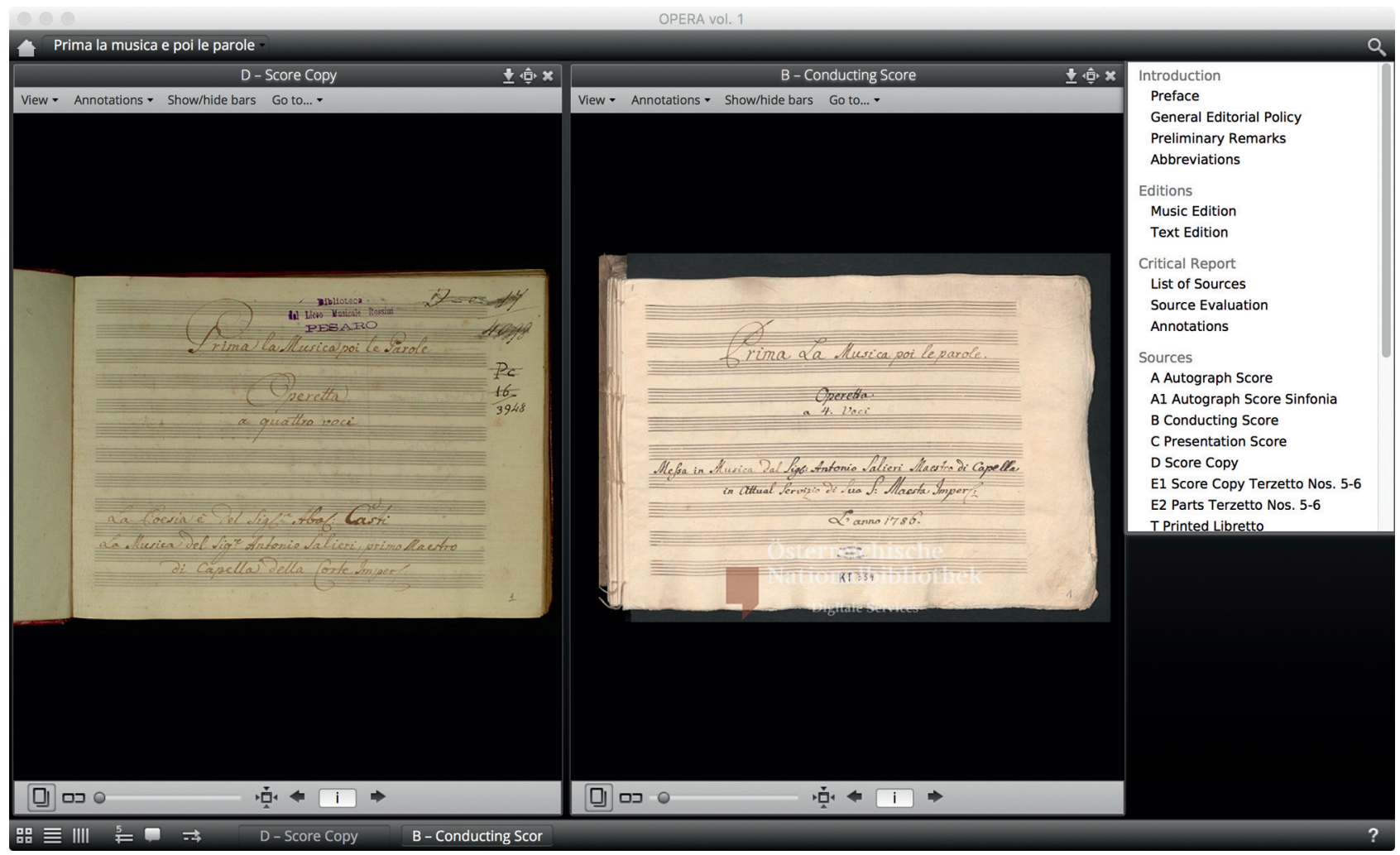

Abb. 2: OPERA vol. 1, Screenshot (Sources: D und Sources: B)

Der in Abb. 1 gezeigte Katalogeintrag verzeichnet ein Medium des Typs „E-Book“, datiert dieses ins Jahr „[1765]“, wobei das „E-Book“ eine „[Partitur]“-Ausgabe ist, den „Umfang“ einer „Abschrift“ hat, als „Kurzaufnahme einer Musikhandschrift" beschrieben werden kann und im Original, das als „Online-Ausgabe“ reproduziert worden ist, am Standort „SLUB Dresden“ steht. Wohlgemerkt: Es soll überhaupt nicht darum gehen, wie missraten dieses ziemlich beliebig herausgegriffene Beispiel vielleicht sein mag, sondern im Gegenteil darum, dass es gerade in seiner Kuriosität so plastisch auf den Reichtum und die Komplexität des Dickichts der Hybride verweist. Die hybriden Digitalisat-Bibliotheken sind für wissenschaftlich Lesende und Schreibende ja ein Segen. ${ }^{16}$ Gleichwohl ist zu betonen, dass das Lesen/Verstehen eines solchen digitalisierten $\mathrm{Ob}$ -

\footnotetext{
16 In Schmales Worten (ebd.): Die „digitale Verfügbarkeit von Quellen und Forschungen“ erleichtert das „Arbeiten schon allein unter dem Aspekt der Zeitökonomie“, und das bei „,g]rößere[r] Breite beziehungsweise Vollständigkeit beim Material“, oder auch „bei der Anwendung von Methoden und Theorien aus anderen Disziplinen“, so dass „Erkenntnis [...] sich schneller einstellen“ kann - „und sie kann schneller veröffentlicht werden.“
}

jekts zwingend Klarheit darüber voraussetzt, was das Digitalisat ,vorher' war, oder genauer: Was es auch ist. ${ }^{17}$

Eine kleine Anekdote aus der Forschung möge dieses Phänomen illustrieren. Der in Abb. 2 zu sehende Screenshot aus der digitalen OPERA-Edition von Antonio Salieris Prima la musica e poi le parole ${ }^{18}$ zeigt zwei der zeitgenössischen Wiener Partiturhandschriften, die diese im Jahr 1786 uraufgeführte Opera buffa überliefern. Im Zuge der Editionsarbeiten wurde über die fassungsgeschichtliche Zuordnung des links eingeblendeten Manuskripts lange gerätselt. Anlass gaben vor allem einige Auffälligkeiten der Nummerierung, etwa beim Finale, normalerweise Nummer 13 der

17 Das kann schon durch scheinbare Äußerlichkeiten mal schwieriger, mal einfacher sein: Etwa ist ein digitalisiertes ,Buch`sehr viel leichter als ,Buch` zu erkennen, wenn Umschläge, Schmutztitel, Vorsatz mit digitalisiert sind, als wenn der Bildschirmleser - wie es so oft der Fall ist - ohne weitere Erläuterung lediglich nur ein Teildigitalisat vorgeführt (,angeboten') bekommt.

18 Casti, Giambattista; Salieri, Antonio: Prima la musica e poi le parole: Divertimento teatrale in un atto, Operetta a quattro voci. Hg. von Thomas Betzwieser und Adrian La Salvia, Redaktion Christine Siegert. Kassel 2013 (OPERA - Spektrum des europäischen Musiktheaters in Einzeleditionen. Historisch-kritische Hybridausgaben. 1), Printausgabe (Vorwort und Partitur), digitale Vollausgabe (Edirom). Auf die digitale Vollausgabe wird im Folgenden verwiesen mit dem Kürzel OPERA vol. 1 . 


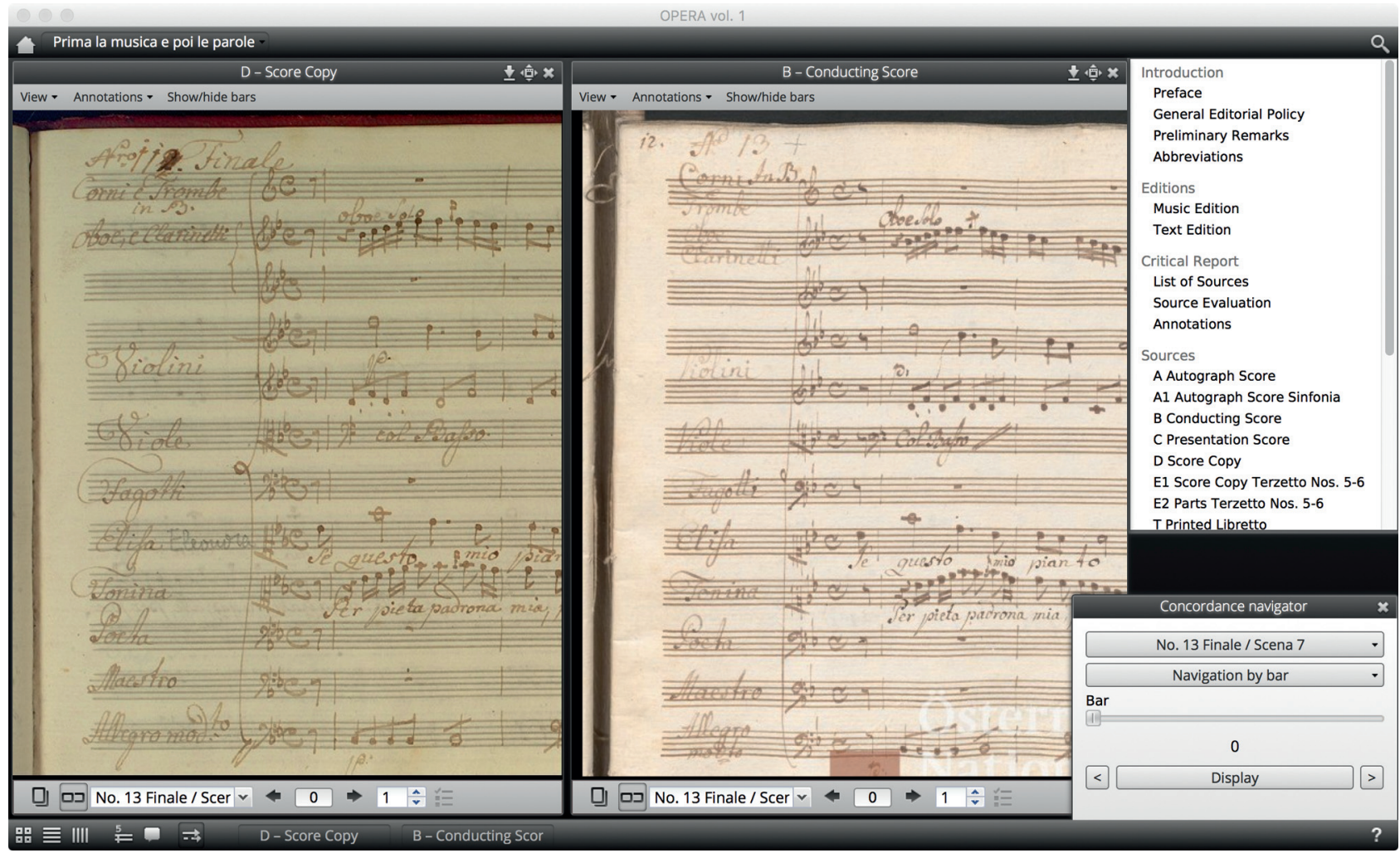

Abb. 3: OPERA vol. 1, Screenshot (Sources: D und Sources: B, Nr. 13)

Oper, in dieser Abschrift aber zunächst als „Nr. 12“ bezeichnet, dann sogar zu „Nr. 11“ abgeändert (siehe Abb. 3).

Für welche gekürzte Aufführung diente also diese Dirigierpartitur? Erst nach ziemlich langer Zeit und erst durch einen Blick auf das Original und seine Abmessungen wurde schlagartig klar: völlig falsche Frage! Denn Quelle D (links) ist - ganz im Gegensatz zur am Bildschirm täuschend ähnlich anmutenden Quelle B (rechts) - überhaupt keine Dirigierpartitur, sondern vielmehr ein bibliophiles Sammelobjekt im Taschenformat (lediglich ungefähr A5, 14x19,5 cm), ganz im Gegensatz zur in Wirklichkeit mehr als doppelt so großen rechten Partitur (22,8x32,8 cm). Die Täuschung der zunächst allzu direkt für das Objekt selbst genommenen digitalen Bilder - oder anders herum: die zunächst nicht hinreichende Einsicht in deren Hybridität - wirkte also als eine durchaus ernstzunehmende methodische Falle.

So interpretiert zeigt die Anekdote, wie entscheidend die Möglichkeit zu wissenschaftlich kompetenter Nutzung davon abhängt, wie gut der Leser im Digitalisat das kulturelle Objekt mitzudenken im Stande ist. Etwas zugespitzter formuliert: Eigentlich verstehen entsprechend kompetent lesende Kulturwissenschaftler Digitalisate überhaupt nur deshalb, weil sie oft genug Originale in der Hand hatten. Und man versteht auch die sog. „Digitalen Sammlungen" umso besser, je mehr man sich in den zugrundeliegenden „Sammlungen“ auskennt.
Wie kaum ein anderer Bibliothekstypus ist die Musikbibliothek schon als physische Bibliothek von ganz besonderem Artenreichtum betroffen. Für das Dickicht der hybriden Musikbibliothek gilt das mithin erst recht: Es ist voller E-Books, E-Papers, E-Journals (übrigens Begriffe, die die Hybridität hervorragend klarstellen - selbst dann noch, wenn mit solchen Begriffen genuin und digitale Publikationen bezeichnet werden, bei denen bestimmte Traditionen der Papier-Welt aber offenbar mitgedacht werden sollen), aber auch voller ,E-Scores', ,E-Manuscripts‘, ,ERecords', ,E-Schellacks‘, ,E-Magnetic-Tapes‘, ,E-PianolaRolls‘. All diese Begriffe gibt es zwar (bisher) nicht, all diese hybriden Spezies aber sehr wohl!

Zuverlässig mitten hinein in diesen Bereich des digitalen Dickichts führt etwa die „Europeana Sounds Collection“. Hier ist u.a. das „Item“ Arnold Schönberg spricht 1931 über das Wagnis moderner Musik verzeichnet (siehe Abb.4). Der „Contributor“ Schönberg spreche, so erfährt man, zugleich auf einem „Tonband auf Kern (AEG)“ und in einer „Mp3-Audiodatei“, und zwar am „1931.03.22“, d.h. im „20. Jahrhundert - 30er Jahre“, in „Wien“ im „Arnold Schönberg Center“ (das wohlgemerkt 1998 eingerichtet wurde).

Angesichts so widersprüchlicher und verwirrender Information über das digitalisierte historische Klangdokument wird man vielleicht mehr herausfinden wollen und 


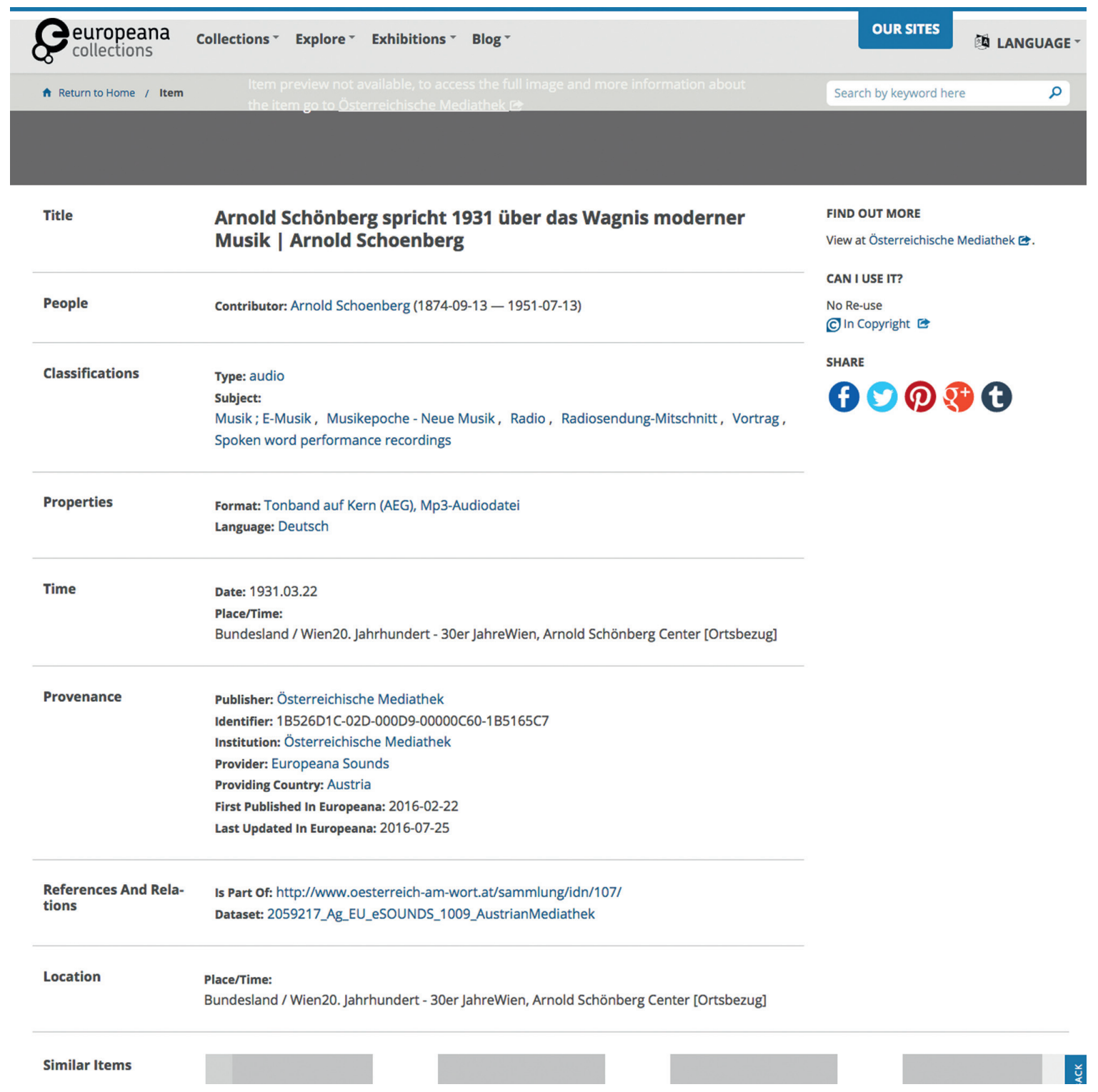

Abb. 4: http://www.europeana.eu/portal/en/record/2059217/data_sounds_1B526D1C_02D_000D9_00000C60_1B5165C7.html, Screenshot (Ausschnitt, 28.8.2017)

daher dem angebotenen Link „View [!] at Österreichische Mediathek" folgen. In der Tat findet man dort weitaus differenziertere Angaben: Ein „Katalogzettel“ unterscheidet hier klar zwischen „Archivformat“ (,Tonband auf Kern (AEG)“) und „Medienart“ (,Mp3-Audiodatei“). Das Datum sei als „[Sendedatum]“ aufzufassen, auch eines der „Schlagworte“ lautet entsprechend „Radiosendung-Mitschnitt“. Doch Moment: Tonband, 1931? Erst 1935 wurde als Weltneuheit das erste AEG Magnetophon auf der Berliner Funkausstellung präsentiert! Solche Rätsel wecken das Verlangen nach „Information“: Der entsprechende Tab führt zunächst zwar nur zu einem Text über den „Inhalt“ (der aus je einem Absatz über die Leidenschaftlichkeit von Schönbergs Plädoyer und über das Profil des Arnold
Schönberg Centers besteht), leitet ganz unten aber in ein Popup-Fenster weiter, das hilfreiche Informationen über die „Sammlungsgeschichte“ bietet: ${ }^{19}$

„Die Sammlung früher Tonaufnahmen der Österreichischen Mediathek enthält einige hundert historische Original-Töne (O-Töne), die aus der Zeit vor der Gründung der Phonothek im Jahr 1960 stammen. [...] Die Aufnahmen stammen von unterschiedli-

19 Vgl. Schaller, Martin: Arbeiten mit digital(isiert)en Quellen: Herausforderungen und Chancen. In: Schmale (2015) (Anm. 15) 24f.: „Die Gedächtnisinstitution an sich ist sortiert. Es steckt eine Geschichte hinter der Sammlung, hinter der Institution. Diese Geschichte wird auch durch die Digitalisierung abgebildet - denn es kann nur digitalisiert werden, was bereits im Bestand ist.“ 


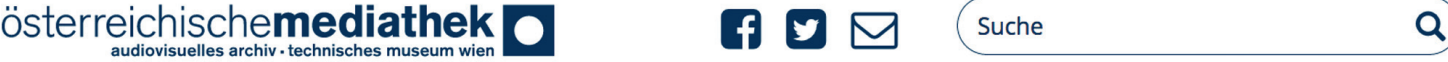

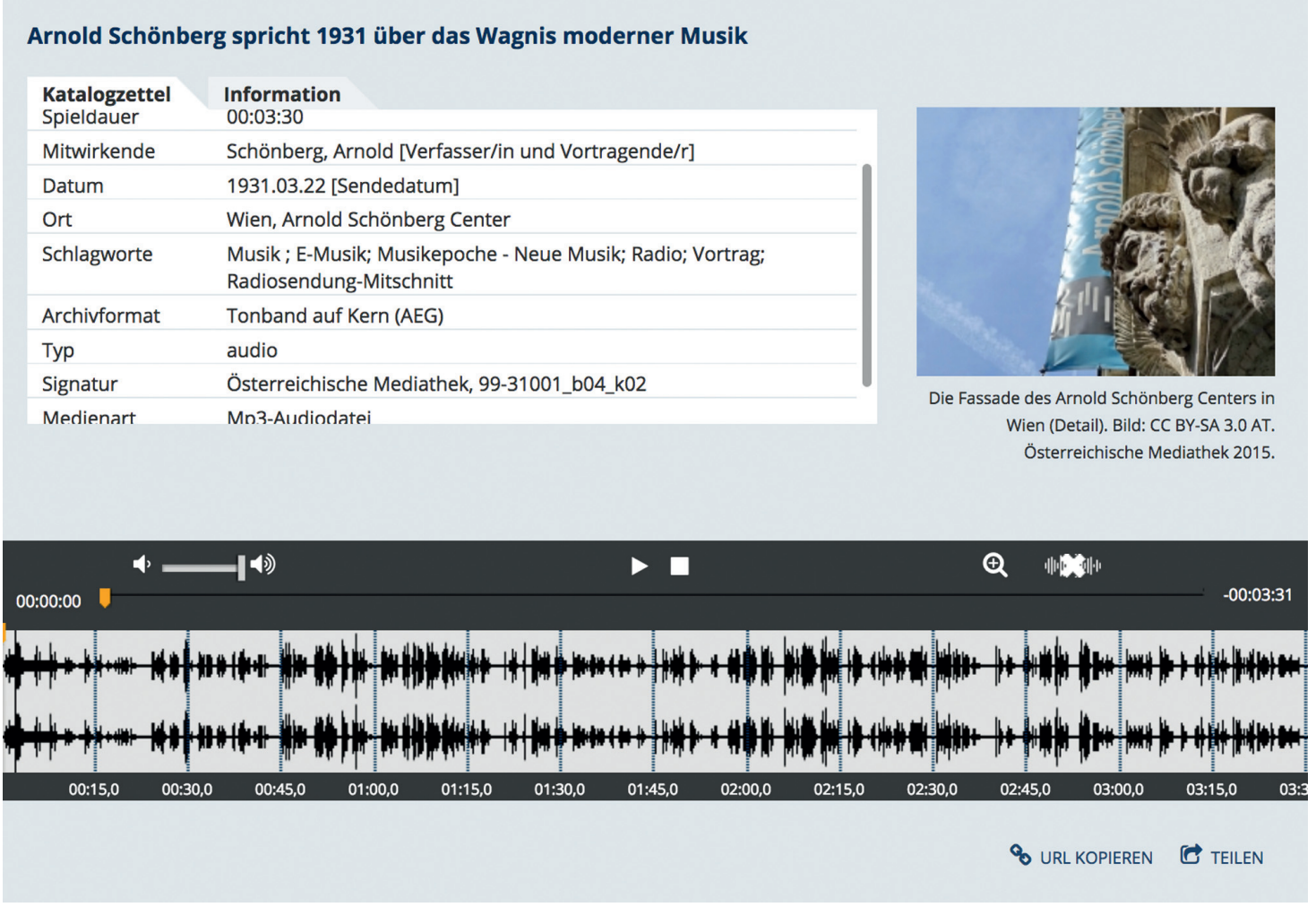

Abb. 5: http://www.mediathek.at/atom/1B526D1C-02D-000D9-00000C60-1B5165C7, Screenshot (Ausschnitt, 28.8.2017)

chen Trägern, Teile davon wurden auf Tonband kopiert. Inhaltlich besteht die Sammlung aus frühen Sprach- und Musikaufnahmen aus der Frühzeit der Tonaufzeichnung [...]. Für die Digitalisierung wurden in diesem Fall die Tonbandaufnahmen als Basis herangezogen, da in den meisten Fällen keine Originalträger mehr verfügbar oder zugänglich sind.“20

Wenn Thomas Walach in einem Beitrag über „Audioquellen als digitale Objekte“ anmahnt, der „permanente und rasche Wandel technischer Aspekte stelle ein „ständiges Problem“ dar, wobei man nicht auf einen „definitiven Endpunkt“ hoffen dürfe, sondern sich „lieber auf eine konstante Evolution von Speichertechnologien einstellen“ sollte, möchte man also beipflichten: Ja, aber das gilt nicht erst für digitale Audioobjekte, sondern schon seit 100 Jahren. Die Mediengeschichte kulturhistorischer Klangdaten wurde nicht durch die Erfindung des Tonbands und erst recht nicht durch die Digitalisierung plötzlich ,auf null ${ }^{*}$ gestellt, auch wenn man als Leser/Hörer im digitalen Archivdickicht leider manchmal - wenn Quellen jeden Alters

20 https://www.mediathek.at/atom/1B526D1C-02D-000D9-00000 C60-1B5165C7 (28.8.2017). und jedweder Art in irgendeiner digital neuberechneten und komprimierten Form in uniformen Playern ,erscheinen' - diesen Eindruck haben könnte. Die Missachtung der medienhistorischen und bestandsgeschichtlichen Dimension erschwert ein medien- und kulturhistorisch kompetentes ,Lesen“ historischer Klangdaten ungemein: In welchem Kontext und auf welches Speichermedium Schönberg wirklich sprach, welche Kopier-, Rekodierungs- und Bearbeitungsroutinen diesem ,audio“ (und „Sound“, wie Europeana klassifiziert, und „Mitschnitt“, wie der Katalogzettel mit einer für diese frühe Zeit der Tonaufnahme eher anachronistischen Vokabel verschlagwortet) inzwischen sukzessive eingeschrieben worden sind, wird allenfalls andeutungsweise mitgeteilt. Die präzisesten Auskünfte sind noch die zugleich am besten versteckten, nämlich die Hinweise auf das ,in den meisten Fällen“ (auch Schönberg?) verschollene Original, auf die Tonbandkopie von ca. 1960 (schon das verzeichnete AEGTonband oder eine weitere Vorstufe?) und die zum Schlagwort degradierte Klassifizierung als Rundfunksendung.

These 2: Gerade im digitalen Dickicht der hybriden Musikbibliothek setzt wissenschaftlich belastbares Lesen ein ganz besonders hohes Maß an medien- und bestands- 
historischem Erfahrungs- und Kontextwissen voraus. Und für Digital Humanities ist das ,Lesen“ im Dickicht nicht etwa einfacher. Denn auch, wenn man den Computer ,lesen' lässt, müssen alle Beteiligten (Computer wie Wissenschaftler) stets genau wissen, was da gefunden, zum Korpus aggregiert, gelesen, ausgewertet oder interpretiert wird. Metadaten, die der Hybridität und Komplexität etwa von Digitalisaten präzise und mit derselben Verlässlichkeit, wie sie für herkömmliche Katalogisierungsroutinen gewohnter Standard ist, gerecht werden, sind für menschliche wie maschinengestütze Lektüren kulturhistorischer Überlieferungen gleichermaßen wichtig.

\section{Noch hybridere Hybride schreiben - eine Hauptbeschäftigung von Digital Humanities}

Digital Humanities, wenn sie kulturhistorische Artefakte und Daten, Beobachtungen und Argumentationen modellieren, vernetzen, visualisieren, ${ }^{21}$ schreiben Hybride: Gebilde, in denen historisches Quellenmaterial, herkömmliche Texte, Kodierungen, Verlinkungen, Programmierungen und Medienumgebungen (Lese-Settings) in einem neuen Ganzen zusammenfließen.

Ein Beispiel sei meiner eigenen Arbeit entnommen; es geht um die historisch-kritische Edition von Justine Favarts Musikkomödie Annette et Lubin aus dem Jahr 1762, bestehend aus einer herkömmlich papierenen Partituredition und einem Datenträger (Edirom). Der (problemlos in jede Musikbibliothek einstellbare) Band ist allerdings nicht ,die Edition', sondern lediglich eine, bestimmten praktischen Rücksichten gehorchende, auszugsweise Ansicht der Edition..$^{22}$ Für die wissenschaftliche (Musik-)Bibliothek müsste also der Leinenband von sekundärer, die EdiromDaten hingegen von primärer Relevanz sein. Wollte man ein wirkliches Inhaltsverzeichnis dessen angeben, was auf den Datenträger geschrieben wurde (und es wäre im Übri-

21 So der Titel der Dhd-Tagung Leipzig 2016: Modellierung, Vernetzung, Visualisierung: Digital Humanities als fächerübergreifendes Forschungsparadigma.

22 Favart, Justine; Blaise, Adolphe: Annette et Lubin. Comédie en un acte en vers, mêlée d'ariettes et de vaudevilles. Hg. von Andreas Münzmay in Zusammenarbeit mit OPERA, supervising editor Janine Droese. Kassel 2016 (OPERA - Spektrum des europäischen Musiktheaters in Einzeleditionen. Historisch-kritische Hybridausgaben 2), Printausgabe (Vorwort und Partitur), digitale Vollausgabe (Edirom). gen höchst erstrebenswert, DH-Forschungsarbeiten künftig mit echten Inhaltsverzeichnissen auszustatten, die das darin Geschriebene vollständig, gerade auch in seinen digitalen Dimensionen, explizit benennen, nicht zuletzt auch deshalb, weil es für eine wirklich adäquate bibliografische Verzeichnung von DH-Publikationen enorm förderlich wäre), hätte dieses sinngemäß folgende Hauptpunkte:

- Bilder (JPG) aller editorisch relevanten Quellen (Kopien von Bibliotheksdigitalisaten, Kopien von selbst hergestellten Fotos) sowie Bilder (JPG) der Musikedition

- und ein Semantisches Markup desselben im Sinne einer Kartografierung (sog. Edirom-,,Vertaktung“: menschengeschriebene/-entschiedene, maschinenlesbare MEI-XML-Kodierung der einzeln gezeichneten und beschrifteten Positionsrahmen),

- Metadaten (insbes. Quellenbeschreibungen, also Metadaten der mit den Bildern ,gemeinten` historischen Artefakte ${ }^{23}$ )

- und ein Semantisches Markup desselben (TEI-XMLKodierung, menschengeschrieben, maschinenlesbar),

- das Lesartenverzeichnis

- und ein Semantisches Markup derselben (TEI-XMLKodierung, erlaubt insbesondere auch den maschinellen Rückbezug jeder Einzellesart auf die betreffenden Stellen der Quellenbilder und der Edition),

- die Librettoedition

- und ein Semantisches Markup derselben (TEI-XMLKodierung nach TEI-Modul 5 Drama),

- eine etwa 3000 Kode-Zeilen umfassende (in jedem Einzeleintrag editorisch entschiedene) Linkliste (MEIXML, menschengeschrieben/-entschieden, maschinenlesbar), mit welcher sich

- ein Konkordanznavigator betreiben lässt (eine digitale Maschine, die jede Stelle des Werks in allen Editionsansichten und Quellendigitalisaten synchron auffindet und ansteuert),

- das Herausgeber-Vorwort samt Semantischem Markup (TEI-XML),

- ein historischer Kommentarapparat zu den im Werk vorkommenden Musik- und Textzitaten samt Semantischem Markup (TEI-XML) und Bildbelegen.

Typisch an diesem digitalen Schreibergebnis sind mithin die „Kernelemente“ geisteswissenschaftlicher „Digitalität“, die Schmale so bündig benannt hat: „Rationalisie-

23 Metadaten der Digitalisate selbst sind - über Bildnachweise hinaus - allerdings leider nicht explizit berichtet, etwa Dateiformate, Bildabmessungen/Auflösungen oder Aufnahmedaten. 
rung, Verflüssigung, Entgrenzung, Dekontextualisierung [,] Personalisierung sowie [...] Veränderungspotenzial“. ${ }^{24}$ Typisch ist aber auch: Hybridität, welche daraus resultiert, dass das ,Material' direkt als Forschungsdaten in die Forschungspublikation mit eingebunden ist. Dieses Miteinbinden aber ist ein systematischer Kardinalunterschied von $\mathrm{DH}$ zu non-DH, zu deren Methodenrepertoire die extensive Möglichkeit, ,das Material zu be-schreiben', ,auf ihm weiterzuschreiben', es elektronisch ,wiederzuschreiben' (vulgo: digitalisieren), um es dann gleichsam , mit Wissenschaft zu beschriften', nicht gehörte. ${ }^{25}$

Eine solche (Weiter-)Hybridisierung von (ohnehin hybridem) Material passiert auch und erst recht, wenn in DHArbeiten Audiomedien involviert sind. Als ein SchreibErgebnis des Projekts Freischütz Digital kann unter http:// freischuetz-digital.de/demos/syncPlayer/test/syncPlayer. xhtml (s. Abb. 6) durch Klick auf „No. 6“, dann auf „Kleiber, Carlos“ eine unter diesem Dirigenten 1973 gemachte Aufnahme des Duetts „Schelm, halt fest!“ aus Webers Freischütz gestartet werden: ein ausgehend von der analogen Schallplatte mehrfach re-kodiertes historisches Artefakt, das irgendwann ins digitale CD-DA-Format übersetzt, dann vielleicht als WAV oder AIFF auf eine Festplatte geschrieben wurde und jetzt eine MP3-Audiodatei ${ }^{26}$ ist - und doch trotz allem auch noch ,die Schallplatte von 1973‘, also das Resultat der Studioproduktion des VEB Deutsche Schallplatten in der erst kurz zuvor zum „Studio Lukaskirche“ umgebauten Dresdner Lukaskirche. ${ }^{27}$

Dieses hybride Objekt hat das Freischütz-Digital-Projekt wiederveröffentlicht und dabei digital ,beschriftet", beispielsweise mit Adressdaten, die eine punktgenaue Verbindung mit MEI-Partitureditionsdaten erlauben (die wiederum mit der Verovio-Bibliothek visualisiert werden).

Digitale ,Beschriftungen' anderer eingebundener Audioquellen-Digitalisate nach identischem Datenmodell ermöglichen natürlich Vergleiche. So kann man - um nur ein Beispiel anzudeuten - Carlos Kleibers Einspielung hö-

24 Schmale, Wolfgang: Digitale Vernunft. In: Historische Mitteilungen 236 (2013/2014), 94.

25 Ein Paradebeispiel für solche digitalen Schreibarbeiten ist die digitale Schaffensprozessforschung im Projekt "Beethovens Werkstatt“; vgl. die unter http://beethovens-werkstatt.de/ publizierten Fallstudien.

$26192 \mathrm{kbps}$, joint stereo, prozessiert mit der Software „Studio One 3.1.0.35191“ - mit ein wenig Spürsinn lassen sich der Datei zumindest ein paar konstitutive Daten entlocken.

27 Besonders umfassende Informationen zu diesem 3 LPs umfassenden Freischütz-Album (DG 2720 071) liefert https://www.discogs.co m/Carl-Maria-von-Weber-Gundula-Janowitz-Edith-Mathis-Peter-Schr eier-Theo-Adam-Staatskapelle-Dresden-Ca/release/3400979 (28.8.2017) ren, dabei aber anhand der synchron angezeigten Timecodes der anderen Aufnahmen gleichzeitig sehen, dass Marek Janowski, 20 Jahre nach Kleiber, zunächst ungefähr gleich schnell musizierte wie Kleiber, ab einem bestimmten Punkt (T. $106 \cong$ Kleiber 3'18" ^ Janowski 3'06”) jedoch signifikant weniger Zeit für dieselbe Menge Musik brauchte, also schneller musizieren ließ. Dieses Lese-Ergebnis bestätigt sich beim Hören: Bei Ännchens Einsatz „Grillen sind mir böse Gäste“ zieht in der Janowski-Produktion plötzlich das Tempo an. Genaues Hinhören identifiziert schließlich auch die Autorin dieser Tempomodifikation, die nicht vom Dirigenten, sondern vielmehr von der Sängerin des Ännchen, Ruth Ziesack, auszugehen scheint: Ungeachtet des beibehaltenen Tempos des Orchesters und der Sängerkollegin Sharon Sweet als Agathe ,zieht` Ruth Ziesack deutlich, so dass es rhythmisch v. a. im dritten und vierten Takt dieser Stelle vernehmlich ,klappert‘. Warum kann gerade dieser Interpretationsforschungsbefund gerade hier herausgelesen werden? Die Antwort ist einfach: Weil er in gerade dieses digitale Datenarrangement gleichsam hineingeschrieben worden ist. Will man den kleinen multimedialen Lesebefund jedoch weiter interpretieren, endet rasch die Aussagekraft der miteinander, mit Quellenbildern und mit MEI-Notentext verlinkten MP3-Dateien, und es kommt die hybride Natur dieser Digitalisate ins Spiel: Wichtig ist etwa, dass es sich bei den mit den Digitalisaten gemeinten Produktionen in beiden Fällen, Carlos Kleiber wie Marek Janowski, um Studioproduktionen handelt (analog im Falle Kleiber, digital im Falle Janowski); das heißt, auch bei Janowski handelt es sich nicht, wie man aufgrund der ,Unsauberkeit‘ der betreffenden Stelle leicht vermuten könnte, um einen Live-Mitschnitt. Dass Dirigent und Produktionsteam die Auffälligkeit schlicht überhörten, ist sehr unwahrscheinlich; sie schrieben es also, aus welchen Gründen auch immer, bewusst so in die CD-Masterdatei.

These 3: Auch die Erzeugnisse digitalen Schreibens sind keineswegs bloß „Informationen“ (so eingebürgert dieses Passepartout-Wort im digitalen Bibliotheksalltag mittlerweile auch sein mag), sondern im Ernst und sogar erst recht Texte. (Allein schon der informatische Schritt der Datenmodellierung ${ }^{28}$ ist ein von Grund auf argumentativer Vorgang, zumal in den Digital Humanities.) Dass die in DH-Zusammenhängen wissenschaftlich geschriebenen (modellierten, vernetzten, visualisierten) musikbezogenen

28 In Wikipedia zurzeit bündig definiert als „Verfahren in der Informatik zur formalen Abbildung der in einem definierten Kontext relevanten Objekte mittels ihrer Attribute und Beziehungen“, vgl. http s://de.wikipedia.org/wiki/Datenmodellierung; Bearbeitungsstand: 7. Mai 2017, 16:32 UTC. 
No. 6 No. 8 No. 9

\section{Recordings}

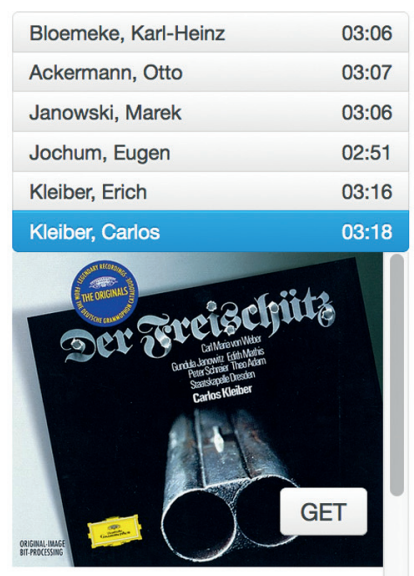

Der

\section{Freischütz}

\section{Conductor}

Kleiber, Carlos

Ensemble

Leipziger Rundfunkchor,

Staatskapelle Dresden

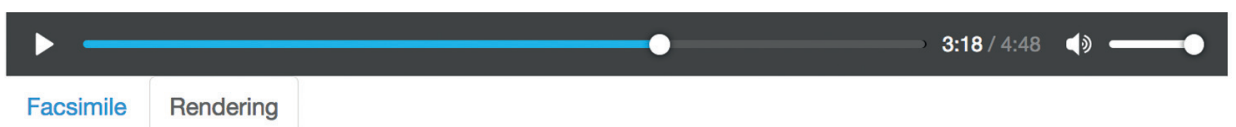

Facsimile Rendering

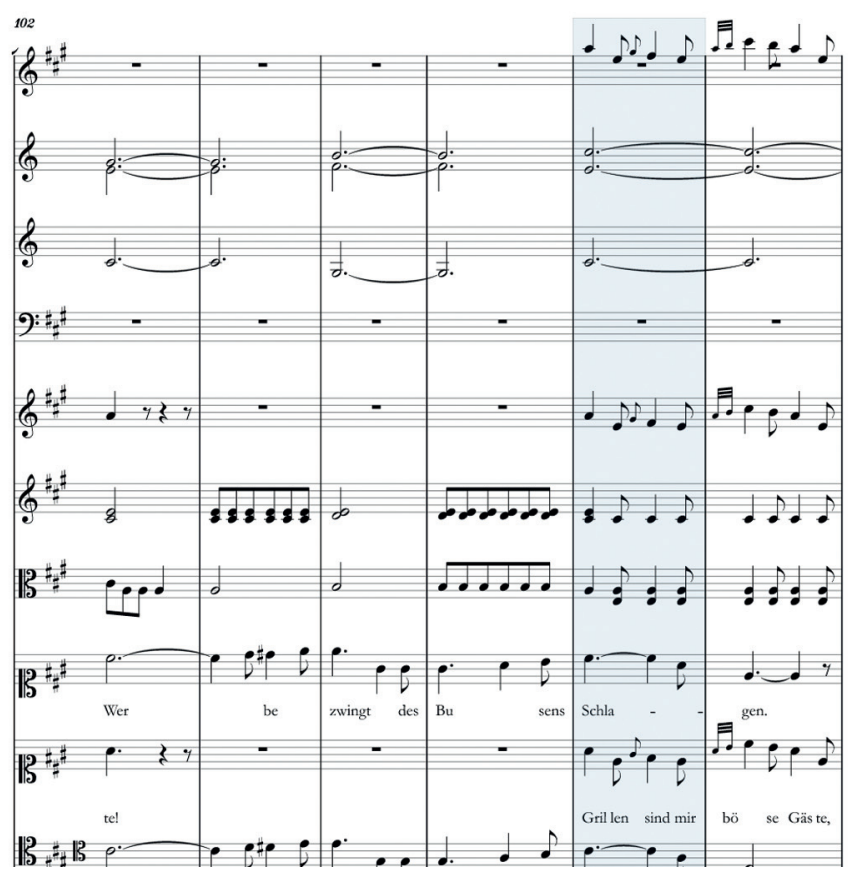

Abb. 6: Freischütz Digital, http://freischuetz-digital.de/demos/syncPlayer/test/syncPlayer.xhtml, Screenshot (Ausschnitt, 28.8.2017)

Daten-Texte musikbibliotheksrelevant sind, steht allein schon deshalb außer Frage. ${ }^{29}$ Es besteht aber auf allen Seiten noch Handlungsbedarf: aufseiten der Wissenschaft, die (noch) zu wenig dafür Sorge trägt, dass ihre digitalen (bzw. in der Regel eben: hybriden) Publikationen überhaupt dezidiert publiziert und dann zudem von wirklich vollständigen, auch die integrierten Re-Publikationen verzeichnenden bibliografischen Daten begleitet werden, und aufseiten der Bibliothek, die (noch) allzu oft kapituliert angesichts des so unübersichtlichen, artenreichen und dynamischen Dickichts digitaler Forschungspublikationen.

29 Oder wie es Nikolaos Beer, Kristin Herold, Peter Stadler und Joachim Veit in einem Positionspapier des Virtuellen Forschungsverbunds Edirom formulierten: „Zu erinnern ist stets daran, dass nicht die (von aktuellen ästhetischen und technischen Einflüssen bedingten) Präsentationen Hauptergebnis von Forschungsprojekten sind, sondern dass deren langfristiger Wert in den erarbeiteten Daten selbst liegt.“ Siehe https://github.com/Edirom/ViFE-website/blob/master/f iles/Positionspapier_ViFE_2016-04.pdf (4.9.2017).

\section{Potenziert hybride Bestände}

Da DH-Texte selbst oft Hybride sind - digitale Be-Schriftungen kulturhistorischen Materials - potenziert sich schlagartig die Hybridität musikbibliothekarischer Bestände, sobald DH-Publikationen dort mit inventarisiert werden. Deshalb ist medienhistorische, bestandsgeschichtliche musikbibliothekarische Kompetenz (die die Objekte auch dann noch durchschaut und für geübte wie ungeübtere Leser transparent zu machen imstande ist, wenn es sich um hybride Objekte/Texte handelt, die andere Objekte/Texte einschließen, die wiederum andere Objekte/Texte einschließen können) gefragter denn je, wenn es darum geht, all die Hybride zum Lesen und weiteren Beforschen bereitzustellen - und wohlgemerkt zunehmend auch Lesern bereitzustellen, die vielleicht noch nie ein entsprechendes Original vor Ohren, vor Augen und in der Hand hatten.

„MP3, 1931“, „Partiturhandschrift, E-Book“ usw.: Die im Zuge von Digitalisierung und Digitalisate-Katalogisierung (noch) so häufig beobachtbare Undeutlichkeit und Unsicherheit geht sicherlich ein Stück weit auf das Konto eines ,Schneller-Höher-Weiter' in einem Wettbewerb um ,umfassendste‘, ,komfortabelste‘ oder ,zentralste‘ Biblio- 
theksdienstleistungen. Die enormen Produktivkräfte, die etwa von der für die Wissenschaft so ungeheuer nützlichen massenhaften Verfügbarkeit von Digitalisaten u.v.m. ausgehen, sollen dabei überhaupt nicht in Abrede gestellt werden! Gleichwohl sei auch auf die Gefahr hingewiesen, dass die in der Digitalisate-Welt noch auf so breiter Front übliche (in der Buch-Welt hingegen wohl undenkbare) Unschärfe das so interessante, artenreiche digitale Dickicht zur amorphen Masse werden lässt, irreführende Fährten legt und das Einzelne in Unkenntlichkeit verschwimmen lässt. Ein trennscharfes automatisiertes ,Lesen“, ,Verarbeiten', ,Auswerten' durch DH-Computer dürften unscharfe, unsaubere Metadaten mit großer Zuverlässigkeit verhindern. Aber auch eine musikwissenschaftliche Hochschullehre, die den Studierenden (und gerade den sog. Digital Natives) das Lesen von und Schreiben über (meist vordigitale) musikkulturhistorische Überlieferungsträger vermitteln will, hat naturgemäß ein großes Interesse an sachrichtigen, widerspruchsfreien und möglichst unmissverständlichen Katalogangaben. Dies beginnt mit dem Ernstnehmen der Hybrididät des vermeintlich lediglich ,digitalen' Materials: Kulturwissenschaftlich und informationstheoretisch zwingend notwendig ist ein konsequenter, trennscharfer Umgang mit Hybriden. Eine Frage ist: Was ist ein Objekt? (Z.B. ein MP3 mit dem Autor X, Titel Y und Signatur Z.) Ein zweiter Fragenkomplex ist aber: Worauf beruht es, was enthält es, was ist es auch?

Und wenn nicht mehr physisch in den Räumen, sondern ,nur' noch per Internet in der Bibliothek gelesen und geschrieben wird, wird die geballte medientypologische und bestandshistorische Kompetenz der Musikbibliothek nicht etwa unwichtiger, sondern im Gegenteil noch sehr viel wichtiger: Je mehr die „Bestände“ dem Nutzer per Digitalisat ausgehändigt werden, desto größer wird der wissenschaftliche Nutzen, der daraus entsteht, dass wenigstens vor Ort in der Gedächtnisinstitution Bibliothek jemand das fragliche kulturhistorische Objekt noch in die Hand und in Augenschein genommen, daran gerochen, es befühlt, es in seiner eigentlichen Machart und Medialität be-griffen hat. Die hybride Bibliothek ist insofern eine Bibliothek, die ihren aus der Ferne lesenden Leser vor Ort gleichsam vertritt: eine wissenschaftlich verantwortungsvolle Aufgabe, bei der jeder Fehler zwangsläufig die Gefahr von Folgefehlern birgt.

These 4: Die Sicherung der Diskursfähigkeit von Publikationen durch klare Referenzierbarkeit gehört seit jeher zu den wichtigsten durch Bibliotheken besorgten Wissenschaftsdienstleistungen überhaupt; dies ist regelrecht eine ,alte Zukunftsaufgabe', die im Wissens-Irrgarten der alten Universalbibliothek durch konkret räumliche Ordnungen, später durch Meta-Verdatung (Katalogisierung; Verschlag- wortung) bewältigt wurde. So ist, was in der Bibliothek ist, prinzipiell für immer wieder auffindbar, als Entität eindeutig, im Diskurs anschlussfähig. Forschungsarbeiten aller Art ,treffen' sich am gemeinsamen beforschten Gegenstand, an gemeinsam diskutierten anderen Forschungsarbeiten. - Und im „digitalen Dickicht“?

Digitalisate wie DH-Forschungsarbeiten bieten gar nicht so wenig Eindeutigkeit, sobald man ihre hybride Natur vorbehaltlos ernst nimmt. Das digitale Dickicht erfordert idealiter einen Katalog der Hybride, einen gleichsam genetischen und jedenfalls relationalen Katalog, in dem die konkreten kulturhistorischen Objekte in ihren konkreten Sammlungskontexten - also als Unikate - Ankerfunktion haben: Vorhanden ist in Institution $\mathrm{W}$ das Digitalisat X, es zeigt Stimmendruck Y und ist von Machart $Z$. Vorhanden ist in derselben Institution $\mathrm{W}$ auch die Digitale Edition A, sie enthält neben dem Digitalisat B (welches seinerseits an seinem Ort seinen eigenen Katalogeintrag hat), der Partitur C (die ebenfalls ihren eigenen Katalogeintrag hat) auch das Digitalisat X (des Stimmendruck-Exemplars Y), sowie die MP3-Datei D, die auf Audio-CD E beruht, die wiederum eine stereophone Re-Edition der Mono-LP $\mathrm{F}$ ist, die das Werk $\mathrm{G}$ in Form der Interpretation $\mathrm{H}$ enthält. In Archiv $\mathrm{V}$ wiederum sind die digitalen Daten $\mathrm{O}$ der Multimediakünstlerin $\mathrm{P}$ zu Performance $Q$ vorhanden, diese Daten sind zugleich eingebunden in die auf Webseite U publizierten Forschungsdaten R, die außerdem auch die Daten zu den Arbeiten S und T derselben Künstlerin enthalten.

Eine solche (per FRBR-Intensivstnutzung wohl im Prinzip umsetzbare, allerdings eine flächendeckende und differenzierte Erfassung v. a. auch von Item-zu-Item-Relationen voraussetzende!) Grundauffassung von ,digitalem Bestand' konzipiert Bestand zwar als Dickicht, aber als sozusagen selbstbewusstes Dickicht, in dem die Spezies identifiziert, die Individuen adressierbar, die Relationen nachzuvollziehen sind: Auch das Digitale Dickicht würde nachhaltig diskursfähig.

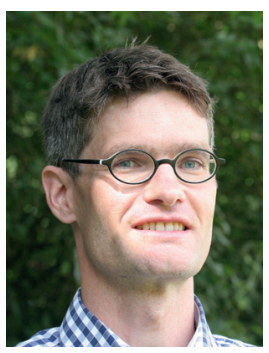

Andreas Münzmay

Universität Paderborn

Musikwissenschaftliches Seminar Detmold/

Paderborn

Hornsche Straße 39

D-32756 Detmold

andreas.muenzmay@uni-paderborn.de 